\title{
On the Nonlinear Dynamics of a Doubly Clamped Microbeam near Primary Resonance
}

\author{
Nizar R. Jaber*, Karim M. Masri^, Mohammad I. Younis*^1 \\ *Physical Science and Engineering Division, King Abdullah University of Science and \\ Technology (KAUST), Thuwal 23955-6900, Saudi Arabia \\ $\wedge$ Department of Mechanical Engineering, State University of New York, Binghamton, NY, \\ 13850, USA
}

\begin{abstract}
This work aims to investigate theoretically and experimentally various nonlinear dynamic behaviors of a doubly clamped microbeam near its primary resonance. Mainly, we investigate the transition behavior from hardening, mixed, and then softening behavior. We show in a single frequency-response curve, under a constant voltage load, the transition from hardening to softening behavior demonstrating the dominance of the quadratic electrostatic nonlinearity over the cubic geometric nonlinearity of the beam as the motion amplitudes becomes large, which may lead eventually to dynamic pull-in.

The microbeam is fabricated using polyimide as a structural layer coated with nickel from top and chromium and gold layers from the bottom. Frequency sweep tests are conducted for different values of DC bias revealing hardening, mixed, and softening behavior of the microbeam. A multi-mode Galerkin model combined with a shooting technique are implemented to generate the frequency response curves and to analyze the stability of the periodic motions using the Floquet theory. The simulated curves show good agreement with the experimental data.
\end{abstract}

\footnotetext{
${ }^{1}$ Corresponding Author: Mohammad I. Younis

e-mail: Mohammad.Younis@kaust.edu.sa
} 


\section{INTRODUCTION}

MEMS resonators are the main building block of many sensors and actuators due to their unique properties of compact size, low power consumption, high sensitivity, high resolution, and the ability to work in harsh environments [1, 2]. They are used in many applications including mass and gas sensing [3-11], efficient energy harvester [12$16]$, and logic gates $[17,18]$.

Electrostatically actuated beams form the heart of many MEMS resonators due to the ease of implementation in electrical circuits, their simple design and fabrication, and their interesting dynamical properties that arise from the interaction between electrostatic, structural, and damping forces. For weakly nonlinear systems, linearized and perturbation approaches might be sufficient. Numerical methods and finite element models need to be implemented for strongly nonlinear systems [2]. Using a relatively simple lumped parameter model, qualitative and quantitative (in some cases) prediction of the resonator response has been presented in [19]. In [20], a comprehensive review of reduced order models of electrostatically actuated beams has been presented. A reduced order model that accounts for the nonlinear electrostatic and restoring forces has been developed and solved for clamped-clamped microbeam using the Galerkin approach [21].

Softening nonlinearity arises if the quadratic nonlinearity from the electrostatic force overcomes the cubic nonlinearity from mid-plane stretching; otherwise, a hardening behavior is reported [2]. Resonators with these nonlinearities have been reported in different types of electrostatically actuated structures including comb drive 
[22, and 23], microplates [24], carbon nanotubes [25], circular membranes [26], and clamped-clamped microbeams [27-29].

Resonators with softening, hardening, and mixed nonlinearities have been proposed for various applications, such as mass sensing [11] and broadband resonators [9]. A tunable comb drive resonator is designed, fabricated, and characterized in [22]. It was showen that by properly selecting the resonator geometry and the operating voltage; the desired nonlinear behavior can be achieved. Thorough numerical analysis of the softening and hardening behavior of the comb drive actuator has been reported in [23]. In [11], an alternative technique is developed for detecting minute masses based on the sudden jumps and bifurcation point of nonlinearly excited MEMS resonators. The nonlinearities and bifurcation points of electrically actuated clamped-clamped microbeams are intensively studied in [30], where the dynamic pull-in phenomenon was studied and proposed for low-voltage MEMS RF switches. The transition from softening to hardening by increasing the laser power of the sensing beam was reported in [29] for clamped-clamped microbeam with a paddle. Also, an analytical model is developed to understand the resonator behavior qualitatively. In [28], a clamped-clamped resonator with tunable nonlinearity is investigated. It was experimentally reported the possibility of five amplitude of vibrations for a given frequency due to the transition from hardening to softening in the same sweep. In [31], an analytical model was developed using five modes in the Galerkin procedure and was solved using the Runge-Kutta algorithm to investigate the resonator response. Although the model predicted the resonator behavior at low amplitudes, it failed to capture the response at high 
amplitude of vibration. In $[32,33]$, it was shown that the shooting method is more robust and can predict the stable and unstable periodic solution accurately compared to Runge-Kutta, which depends on robustness and the size of the basin of attraction.

As shown in the literature, many researchers reported the utilization of the DC voltage to tune the nonlinearities of electrically actuated microbeams. The proposed models however have failed to quantitatively match the simulations results with the nonlinear experimental data; especially at high amplitudes of vibration. This paper aims to present a model and simulation technique, combined with careful experimental data to investigate the nonlinear dynamics of the resonant beam near its primary resonance showing hardening, softening, and mixed behavior near pull-in. An accurate model is developed based on the Galerkin approach combined with the shooting technique that is capable of quantitatively simulating the experimental response is developed.

\section{FABRICATION AND EXPERIMENTAL SETUP}

The clamped-clamped microbeam is fabricated using the process developed in [34], as depicted in Fig. 1. The microstructure consists of a $6 \mu \mathrm{m}$ polyimide structural layer coated with $500 \mathrm{~nm}$ nickel layer from the top and $50 \mathrm{~nm}$ chrome, $250 \mathrm{~nm}$ gold and $50 \mathrm{~nm}$ chrome from the bottom. The lower electrode is placed directly underneath the microstructure and composed of gold and chrome layers. It spans half of the microstructure length and provides the actuation force to the resonator. The half electrode is made to excite the symmetric and anti-symmetric modes of the beam [35]. When the two electrodes connected to an external excitation voltage, the resonator vibrates in the out-of-plane direction of Fig. 2. 


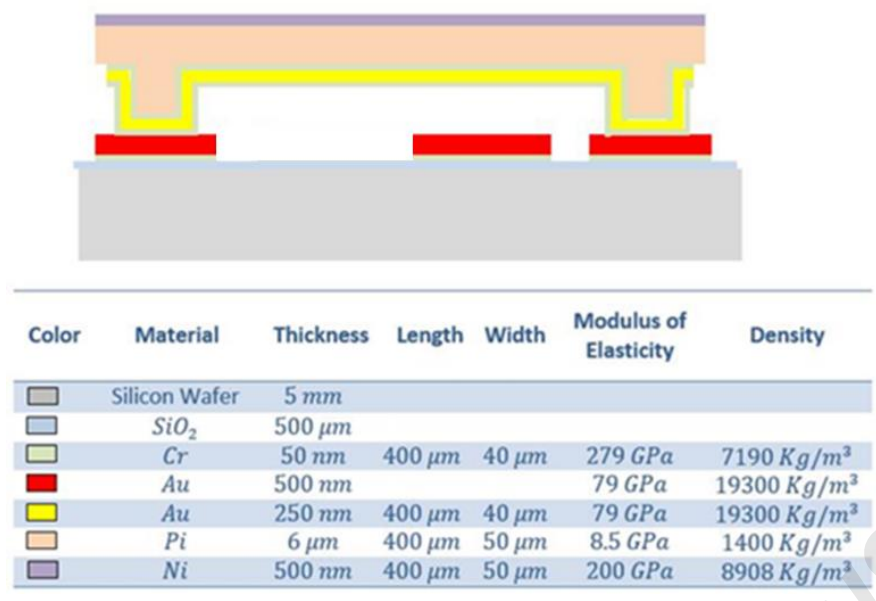

Figure 1: A cross-sectional view of the fabricated microbeam.

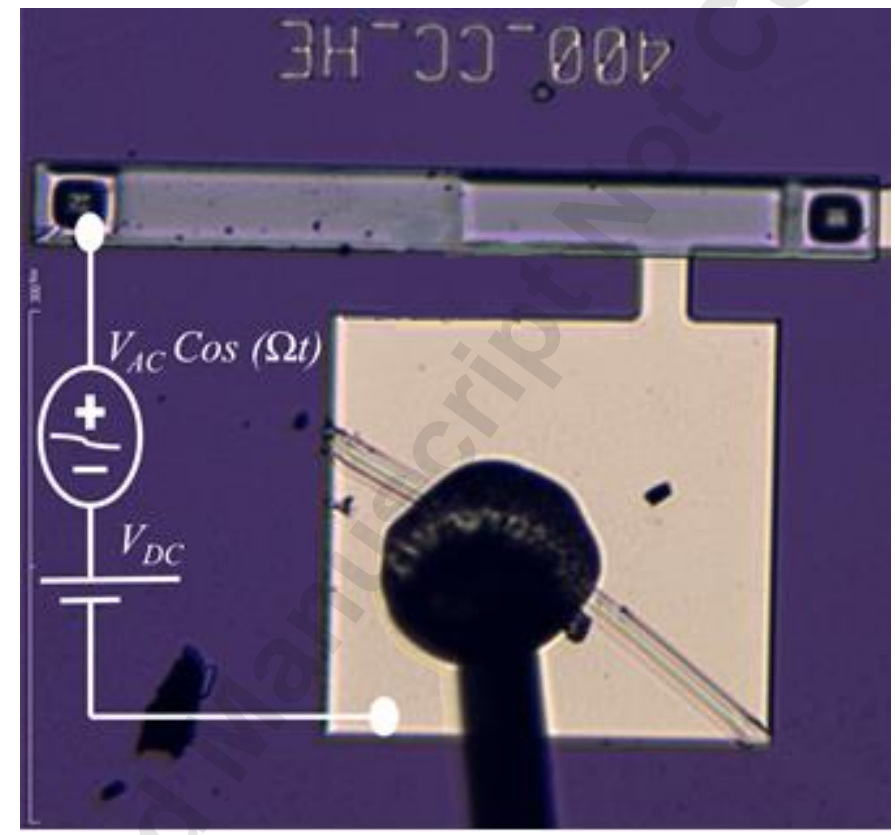

Figure 2: A top view picture of the fabricated microbeam and the actuation pad.

Next, we describe the experimental setup used for testing the device and measuring the initial profile, gap thickness, and the out-of-plane vibration (z-direction). The experimental setup consists of a microsystem analyzer (MSA) under which the microbeam is placed to measure its vibration, data acquisition cards, and amplifier to 
provide actuation signals of a wide range of frequencies and amplitudes, and a vacuum chamber equipped with ports to pass the actuation signal and measure the pressure. Also, it is hooked up to a vacuum pump that reduces the pressure up to 3 mTorr. The microbeam movement is measured using the laser-Doppler vibrometer. The setup is shown in Fig.3.

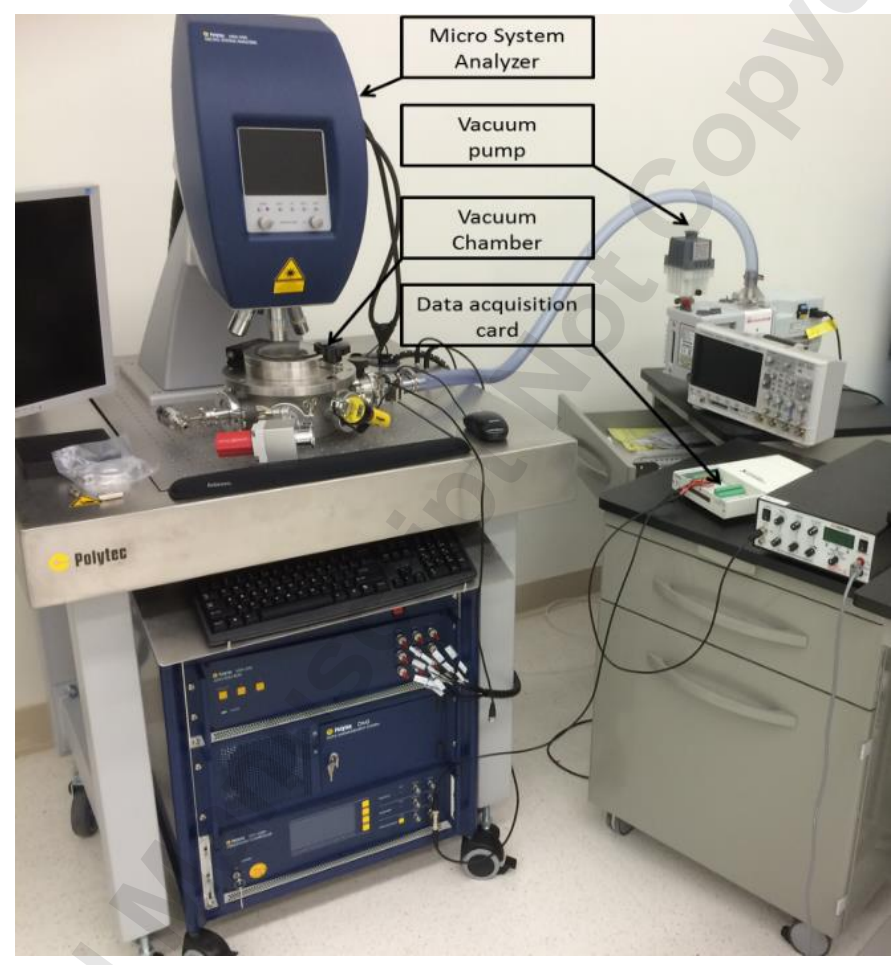

Figure 3: Experimental setup used for testing the MEMS device.

\section{THE MODEL}

The governing equation of motion of the clamped-clamped beam with a half electrode, Fig. 4, which is actuated by the electrical loads $V_{D C}$ and $V_{A C}$ applied on the beam's lower electrode is written as 


$$
\begin{aligned}
& E l \frac{\partial^{4} w}{\partial x^{4}}+\rho A \frac{\partial^{2} w}{\partial t^{2}}+c \frac{\partial w}{\partial x}=\left(N+\frac{E A}{2 \ell} \int_{0}^{\ell}\left(\frac{\partial w}{\partial x}\right)^{2} \partial x\right) \frac{\partial^{2} w}{\partial x^{2}} \\
& +\frac{\varepsilon b\left[V_{D C}+V_{A C} \operatorname{Cos}(\Omega t)\right]^{2}[U(x)-U(x-0.5 \ell)]}{2(d-w)^{2}}
\end{aligned}
$$

where $x$ is the position along the beam, $t$ is time, $E l$ is the effective flexural of rigidity, $\rho$ is the density, $E$ is Young's modulus, $A$ is the cross-section area, $\ell$ is the length, $c$ is the damping coefficient, $N$ is the tensile axial force, $\varepsilon$ is the separation dielectric constant, $\Omega$ is the excitation frequency, $d$ is the separation distance, and $U(x)$ is the unit step function. The boundary conditions for a clamped-clamped beam are

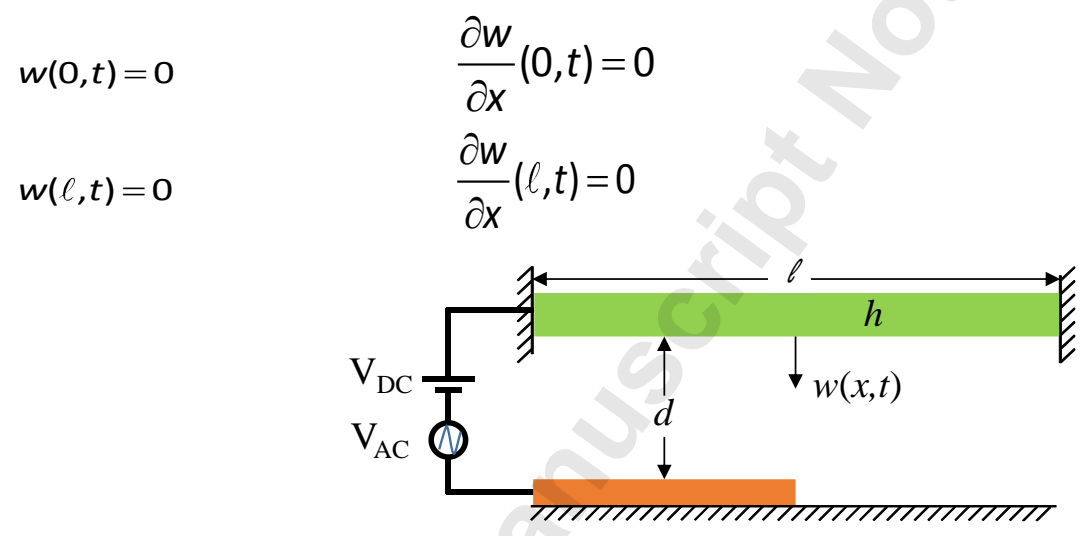

Figure 4: Schematic of the doubly clamped microbeam with the half electrode.

To determine the beam response, we derive a reduced order model ROM using the Galerkin method [2]. Hence, the beam deflection is expressed as

$w(x, t)=\sum_{i=1}^{n} u_{i}(t) \phi_{i}(x)$

where $\phi_{i}(x)$ is the beam's $i^{\text {th }}$ linear undamped unforced orthonormal modeshape, and $u_{i}(t)$ is the $i^{\text {th }}$ generalized modal coordinate. The ROM produces a set of differential equations in terms of the modal coordinates. Three modes were utilized in the Galerkin 
approximation. Then, the shooting method is used to capture the stable and unstable periodic orbits and analyze their stability using the Floquet theory [2]. The use of linear mode shapes significantly reduces the computation time compared with using finite element software to extract the basis function.

One can note that although the used basis functions in the Galerkin method were of linear type (linear mode shapes) the use of these for nonlinear electrostatic MEMS problem has been proven to be accurate provided that enough number of modes are used and convergence is checked. Here, we use three symmetric modes, which was shown to be sufficient for convergence $[2,20,30,37]$.

\section{DEVICE CHARACTERIZATION}

The initial profile of the microstructure is revealed using an optical profilometer, which generates a 3-D map of the microstructure as seen from the top in Fig. 5. The combined thickness of the microstructure and air gap is around $9 \mu m$, which is slightly smaller from the nominal design value of $9.35 \mu \mathrm{m}$. Also, the microbeam profile is uniformly straight without any curvature or curling. 

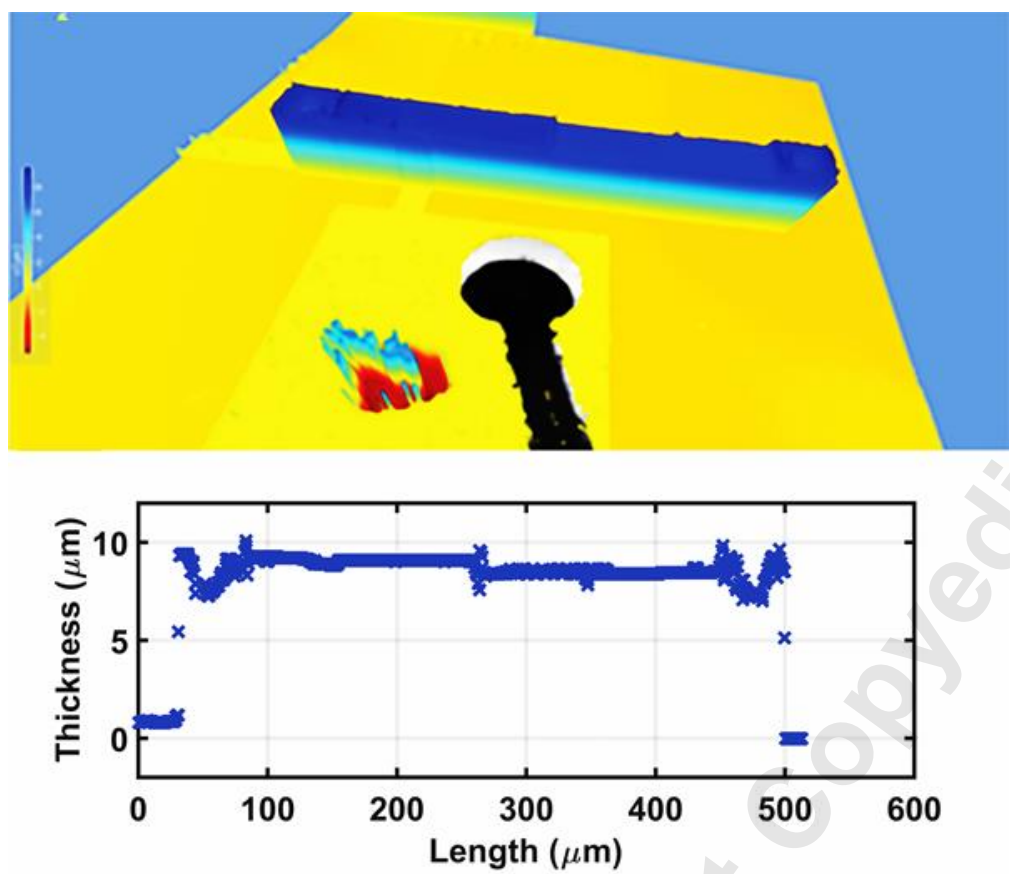

Figure 5: A 3-D map of the microstructure profile as seen from the top.

To experimentally calculate the flexural rigidity $E I$, we initially biased the microstructure by applying a slow $D C$ ramp voltage $\left(V_{D C}\right)$ - generated using the data acquisition card - and measured the static deflection $W$ at half of the beam, Fig. 6 . The deflection increases until it exhibits pull-in at $168 \mathrm{~V}$. Then, the analytical static equation is solved for different $E I$ until the analytical and experimental results are matched.

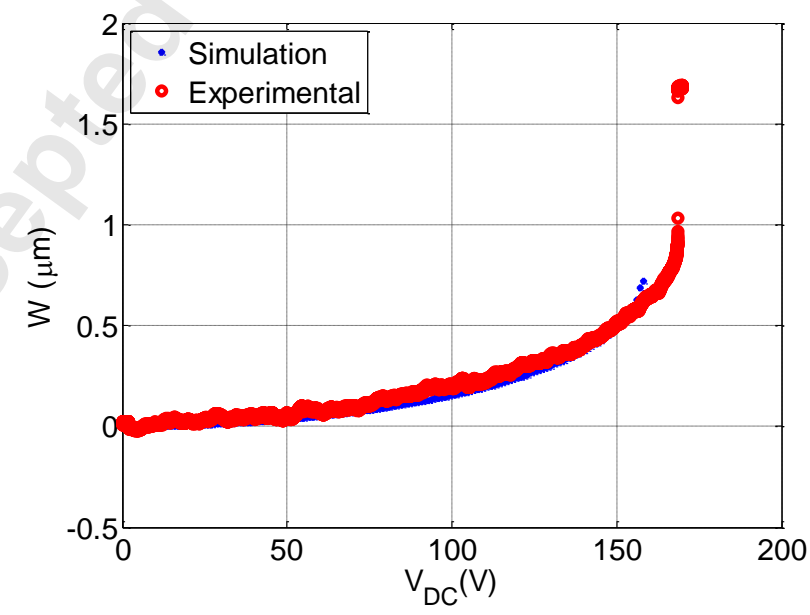


Figure 6: Static deflection of the beam midpoint with the DC voltage until pull-in.

The resonance frequencies values and the corresponding mode shape are revealed by actuating the microbeam with a white noise signal and then scanning the vibration along the beam surface. The acquired frequency response curve is shown in Fig. 7, which reveals the values of the first three natural frequencies $\omega_{1}=160 \mathrm{kHz}, \omega_{2}=$ $402 \mathrm{kHz}$, and $\omega_{3}=738 \mathrm{kHz}$. The velocity RMS values of the test are reported in the insets of Fig. 7. We notice at $\omega_{1}$, all points are vibrating whereas, at $\omega_{2}$, the midpoints are nodal points. Also, at $\omega_{3}$ there are two nodal points. These results match the clampedclamped structure first, second, and third mode shapes of vibration.

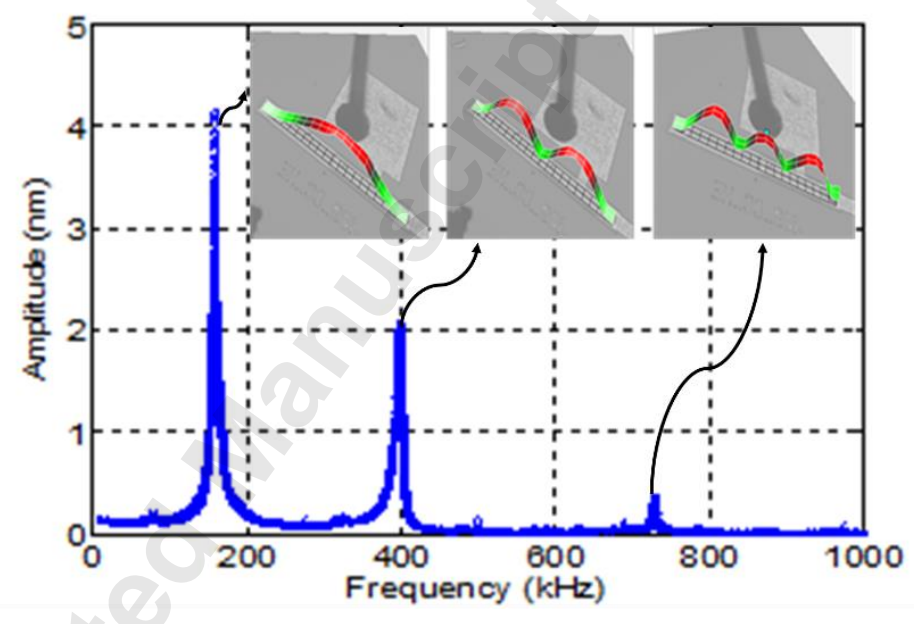

Figure 7: Frequency response to white noise actuation signal at $V_{D C}=30 \mathrm{~V}, V_{A C}=$ $50 \mathrm{~V}$, and at 4 mTorr chamber pressure.

\section{RESULTS}

Next, we show several frequency response curves starting from linear, then hardening, mixed, and totally softening behavior. Both theoretical and experimental 
data will be shown and compared. For the model, the flexural rigidity $E l$ is extracted by investigating the static deflection curve. The theoretical value matches with the experimental results shown in Fig. 6 when $E I=1.12 \times 10^{-10} \mathrm{~N} \cdot \mathrm{m}^{2}$. Figure 8 shows the fundamental natural frequency $f_{n}=160 \mathrm{kHz}$. The quality factor $Q=300$ is extracted from the experimental data depicted in Fig. 8, which will be used in the analytical analysis.

Figure 9 shows the maximum beam deflection when $V_{D C}=25 V$ and $V_{A C}=5 \mathrm{~V}$. The solid line is the stable solution while the red dashed line is unstable. There is a saddle-node bifurcation at $f=160.1 \mathrm{kHz}$ where a Floquet multiplier approaches unity leading to the unstable solution where the beam jumps from one stable branch to the other.

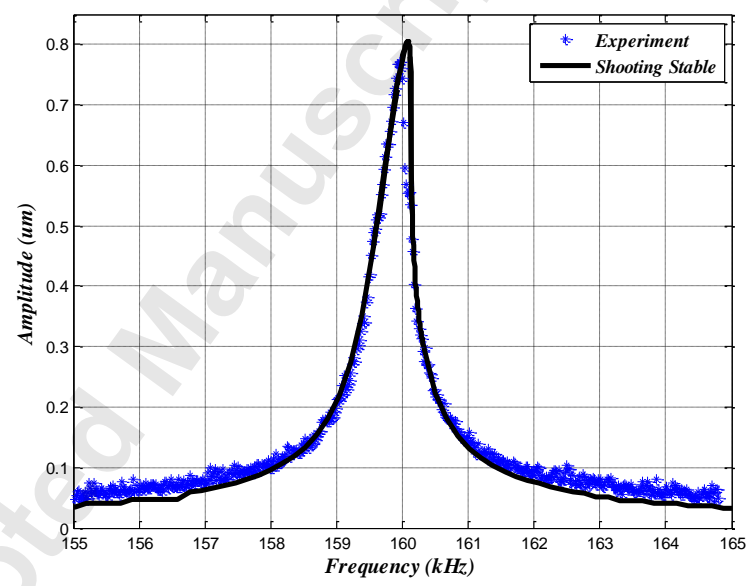

Figure 8: Frequency response curve showing almost linear behavior at $V_{D C}=20$

$V, V_{A C}=5 \mathrm{~V}$. 


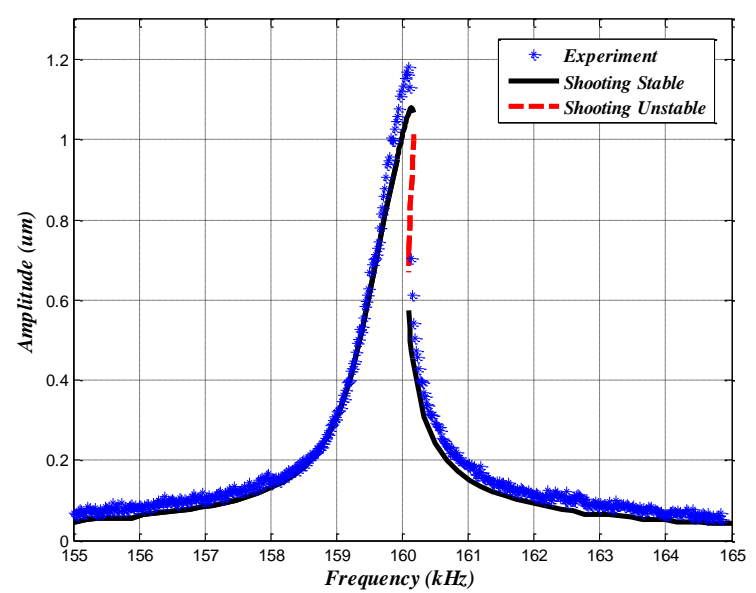

Figure 9: Frequency response curve showing hardening behavior at $V_{D C}=25 \mathrm{~V}$, $V_{A C}=5 \mathrm{~V}$.

Figure 10 showes the variation of the beam deflection when the DC voltage is increased to $V_{D C}=35 \mathrm{~V}$. The figure shows a hardening and then a softening behavior at very large deflection (mixed behavior). In this case; there are three saddle-node bifurcations reported where the stable and unstable solutions meet. First, sweeping the frequency up, at the left branch at $f=159.5 \mathrm{kHz}$ will result in a jump to the stable upper branch or pull-in. Increasing the frequency on the upper right branch to cross the frequency $f=160 \mathrm{kHz}$ will cause a drop in the amplitude in the stable lower branch. Finally, sweeping down on the right lower stable branch at $f=159.9 \mathrm{kHz}$ will lead to a jump to the upper stable branch or pull-in (depending on the strength of the basin of attractions of each attractor [38-41]). 


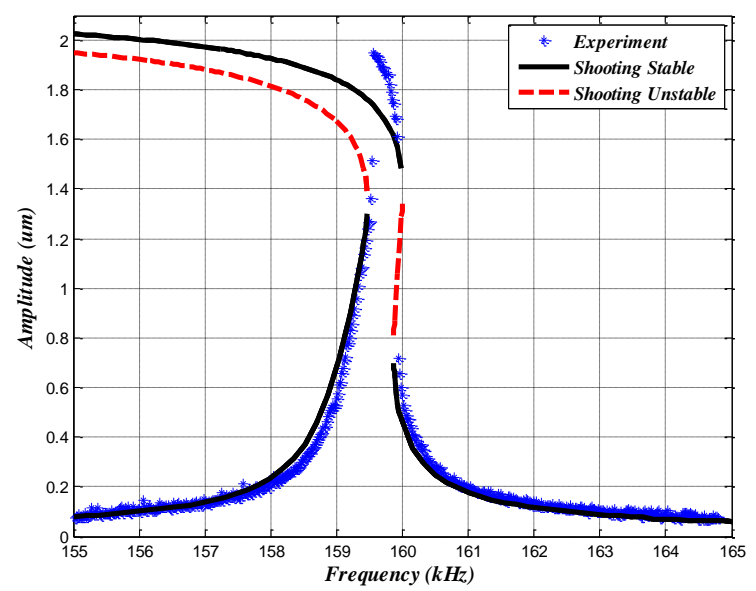

Figure 10: Frequency response curve showing a hardening then softening behavior (mixed response) at $V_{D C}=35 V, V_{A C}=5 \mathrm{~V}$.

Increasing the $D C$ voltage to $V_{D C}=40 V$ and $V_{D C}=45 V$ as shown in Fig. 11 and Fig. 12 , respectively, causes a softening behavior. In Fig.11, increasing the frequency and crossing $f=159 \mathrm{kHz}$ will lead to jumps to the upper stable solution or pull-in. Figure 12 shows a pure softening behavior. At this large voltage load, the quadratic nonlinearity from the electrostatic force completely overcomes the cubic geometric nonlinearity. Hence, the effective nonlinearity of the system even at moderately small vibration amplitude becomes of softening type [21].

Although the model predicted the experimental data for most of the parts, some discrepancies can be noticed especially at high amplitudes. These discrepancies can be attributed to the fabrication imperfections and the irregularities of the microbeam at the midpoint -where the measurement is recorded- due to the half electrode configuration, as depicted in Fig. 2 and Fig. 5. 


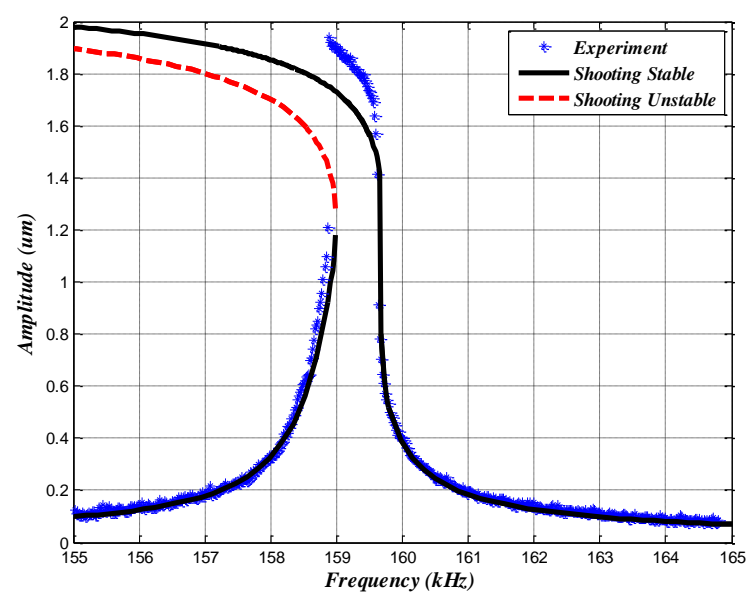

Figure 11: Frequency response curve showing a hardening then softening behavior (mixed response) at $V_{D C}=40 \mathrm{~V}, V_{A C}=5 \mathrm{~V}$.

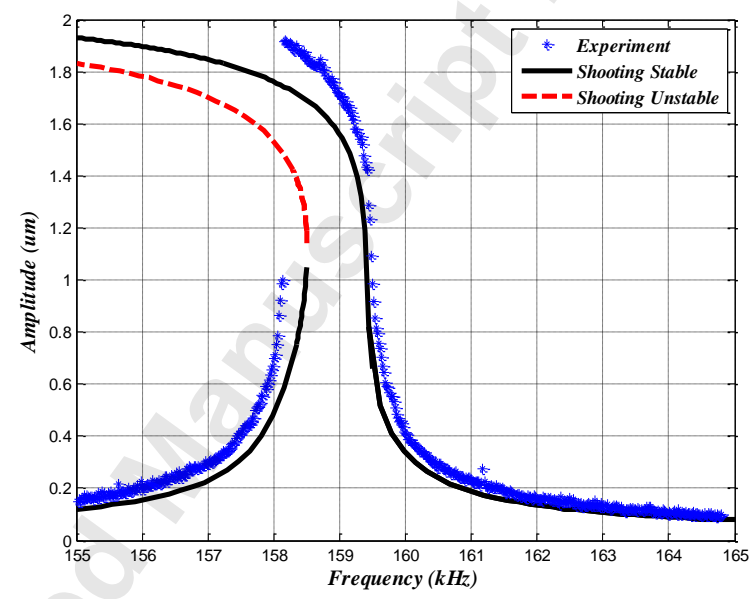

Figure 12: Frequency response curve showing a softening behavior at $V_{D C}=45 \mathrm{~V}$,

$V_{A C}=5 \mathrm{~V}$.

\section{CONCLUSIONS}

We studied the dynamics of the micro doubly clamped beam when it is actuated electrostatically near its fundamental natural frequency. The microbeam is actuated with a lower electrode that covers its half-length. We showed several frequency 
response curves at different values of DC loads demonstrating linear, hardening, mixed (hardening and softening), and pure softening behaviors. The reduced order model along with shooting technique was used to simulate the resulting frequency response curves. We reported experimentally and theoretically the regaining of stability of the unstable branch of the frequency response curve at large amplitude almost near the lower electrode. The simulated results showed good agreement with the experimental data where we noticed a hardening behavior at lower DC voltage and softening and dynamic pull-in at higher DC voltage loads.

\section{REFERENCES}

[1] Zhang, W.-M., Hu, K.-M., Peng, Z.-K., and Meng, G., 2015, "Tunable Micro- and Nanomechanical Resonators," Sensors, 15(10).

[2] Younis, M. I., 2011, MEMS Linear and Nonlinear Statics and Dynamics: Mems Linear and Nonlinear Statics and Dynamics, Springer Science \& Business Media.

[3] Thundat, T., Wachter, E., Sharp, S., and Warmack, R., 1995, "Detection of mercury vapor using resonating microcantilevers," Applied Physics Letters, 66(13), pp. 16951697.

[4] Schmid, S., Senn, P., and Hierold, C., 2008, "Electrostatically actuated nonconductive polymer microresonators in gaseous and aqueous environment," Sensors and Actuators A: Physical, 145, pp. 442-448.

[5] Kumar, V., Boley, J. W., Yang, Y., Ekowaluyo, H., Miller, J. K., Chiu, G. T.-C., and Rhoads, J. F., 2011, "Bifurcation-based mass sensing using piezoelectrically-actuated microcantilevers," Applied Physics Letters, 98(15), p. 153510. 
[6] Dohn, S., Sandberg, R., Svendsen, W., and Boisen, A., 2005, "Enhanced functionality of cantilever based mass sensors using higher modes," Applied Physics Letters, 86(23), p. 233501.

[7] Olcum, S., Cermak, N., Wasserman, S. C., and Manalis, S. R., 2015, "High-speed multiple-mode mass-sensing resolves dynamic nanoscale mass distributions," Nature communications, 6 .

[8] Jin, D., Li, X., Liu, J., Zuo, G., Wang, Y., Liu, M., and Yu, H., 2006, "High-mode resonant piezoresistive cantilever sensors for tens-femtogram resoluble mass sensing in air," Journal of Micromechanics and Microengineering, 16(5), p. 1017.

[9] Cho, H., Yu, M.-F., Vakakis, A. F., Bergman, L. A., and McFarland, D. M., 2010, "Tunable, broadband nonlinear nanomechanical resonator," Nano letters, 10(5), pp. 1793-1798.

[10] Hanay, M., Kelber, S., Naik, A., Chi, D., Hentz, S., Bullard, E., Colinet, E., Duraffourg, L., and Roukes, M., 2012, "Single-protein nanomechanical mass spectrometry in real time," Nature nanotechnology, 7(9), pp. 602-608.

[11] Nguyen, V.-N., Baguet, S., Lamarque, C.-H., and Dufour, R., 2015, "Bifurcation-based micro-/nanoelectromechanical mass detection," Nonlinear Dynamics, 79(1), pp. 647662.

[12] Harne, R., and Wang, K., 2013, "A review of the recent research on vibration energy harvesting via bistable systems," Smart Materials and Structures, 22(2), p. 023001. 
[13] Erturk, A., and Inman, D. J., 2008, "A distributed parameter electromechanical model for cantilevered piezoelectric energy harvesters," Journal of vibration and acoustics, 130(4), p. 041002.

[14] Abdelkefi, A., Najar, F., Nayfeh, A., and Ayed, S. B., 2011, "An energy harvester using piezoelectric cantilever beams undergoing coupled bending-torsion vibrations," Smart Materials and Structures, 20(11), p. 115007.

[15] Abdelkefi, A., Nayfeh, A., Hajj, M., and Najar, F., 2012, "Energy harvesting from a multifrequency response of a tuned bending-torsion system," Smart Materials and Structures, 21(7), p. 075029.

[16] Jemai, A., Najar, F., Chafra, M., and Ounaies, Z., 2016, "Modeling and parametric analysis of a unimorph piezocomposite energy harvester with interdigitated electrodes," Composite Structures, 135, pp. 176-190.

[17] Mahboob, I., Flurin, E., Nishiguchi, K., Fujiwara, A., and Yamaguchi, H., 2011, "Interconnect-free parallel logic circuits in a single mechanical resonator," Nature communications, 2, p. 198.

[18] Hafiz, M. A. A., Kosuru, L., and Younis, M. I., 2016, "Microelectromechanical reprogrammable logic device," Nature communications, 7.

[19] Mestrom, R., Fey, R., Van Beek, J., Phan, K., and Nijmeijer, H., 2008, "Modelling the dynamics of a MEMS resonator: simulations and experiments," Sensors and Actuators A: Physical, 142(1), pp. 306-315.

[20] Nayfeh, A. H., Younis, M. I., and Abdel-Rahman, E. M., 2005, "Reduced-Order Models for MEMS Applications," Nonlinear Dynamics, 41(1), pp. 211-236. 
[21] Younis, M. I., and Nayfeh, A. H., 2003, "A Study of the Nonlinear Response of a Resonant Microbeam to an Electric Actuation," Nonlinear Dynamics, 31(1), pp. 91-117. [22] Rhoads, J. F., Shaw, S. W., Turner, K. L., Moehlis, J., DeMartini, B. E., and Zhang, W., 2006, "Generalized parametric resonance in electrostatically actuated microelectromechanical oscillators," Journal of Sound and Vibration, 296(4), pp. 797829.

[23] Elshurafa, A. M., Khirallah, K., Tawfik, H. H., Emira, A., Aziz, A. K. A., and Sedky, S. M., 2011, "Nonlinear dynamics of spring softening and hardening in folded-MEMS comb drive resonators," Journal of Microelectromechanical Systems, 20(4), pp. 943-958.

[24] Saghir, S., and Younis, M. I., 2016, "An investigation of the static and dynamic behavior of electrically actuated rectangular microplates," International Journal of NonLinear Mechanics, 85, pp. 81-93.

[25] Ouakad, H. M., and Younis, M. I., 2009, "Nonlinear Dynamics of Electrically Actuated Carbon Nanotube Resonators," Journal of Computational and Nonlinear Dynamics, 5(1), pp. 011009-011009.

[26] Kojiro, T., Keiichiro, N., Masao, N., Hiroshi, Y., Shin'ichi, W., and Sunao, I., 2009, "Direct Actuation of GaAs Membrane with the Microprobe of Scanning Probe Microscopy," Japanese Journal of Applied Physics, 48(6S), p. 06FG06.

[27] Mestrom, R., Fey, R., Phan, K., and Nijmeijer, H., 2010, "Simulations and experiments of hardening and softening resonances in a clamped-clamped beam MEMS resonator," Sensors and Actuators A: Physical, 162(2), pp. 225-234. 
[28] Kacem, N., and Hentz, S., 2009, "Bifurcation topology tuning of a mixed behavior in nonlinear micromechanical resonators," Applied Physics Letters, 95(18), p. 183104.

[29] Sahai, T., Bhiladvala, R. B., and Zehnder, A. T., 2007, "Thermomechanical transitions in doubly-clamped micro-oscillators," International Journal of Non-Linear Mechanics, 42(4), pp. 596-607.

[30] Nayfeh, A. H., Younis, M. I., and Abdel-Rahman, E. M., 2007, "Dynamic pull-in phenomenon in MEMS resonators," Nonlinear Dynamics, 48(1), pp. 153-163.

[31] Kacem, N., Hentz, S., Pinto, D., Reig, B., and Nguyen, V., 2009, "Nonlinear dynamics of nanomechanical beam resonators: improving the performance of NEMS-based sensors," Nanotechnology, 20(27), p. 275501.

[32] Bataineh, A., and Younis, M., 2014, "Dynamics of a clamped-clamped microbeam resonator considering fabrication imperfections," Microsystem Technologies, pp. 1-10. [33] Jaber, N., Ramini, A., and Younis, M. I., 2016, "Multifrequency excitation of a clamped-clamped microbeam: Analytical and experimental investigation," Microsystems \& Nanoengineering, 2.

[34] Arevalo, A., Byas, E., Conchouso, D., Castro, D., Ilyas, S., and Foulds, I. G., "A versatile multi-user polyimide surface micromachinning process for MEMS applications," Proc. Nano/Micro Engineered and Molecular Systems (NEMS), 2015 IEEE 10th International Conference on, pp. 561-565.

[35] Jaber, N., Ramini, A., Carreno, A. A., and Younis, M. I., 2016, "Higher order modes excitation of electrostatically actuated clamped-clamped microbeams: experimental 
and analytical investigation," Journal of Micromechanics and Microengineering, 26(2), p. 025008.

[36] Caruntu, D. I., and Knecht, M. W., 2015, "Microelectromechanical systems cantilever resonators under soft alternating current voltage of frequency near natural frequency," Journal of Dynamic Systems, Measurement, and Control, 137(4), p. 041016. [37] Younis, M. I., Abdel-Rahman, E. M., and Nayfeh, A., 2003, "A reduced-order model for electrically actuated microbeam-based MEMS," Journal of Microelectromechanical Systems, 12(5), pp. 672-680.

[38] L. Ruzziconi1, A. H. Ramini, M. I. Younis, and S. Lenci, "Theoretical prediction of experimental jump and pull-in dynamics in a MEMS sensor," Sensors, 14, 17089-17111; doi:10.3390/s140917089, 2014.

[39] L. Ruzziconi, M. I. Younis, and S. Lenci, “Multistability in an electrically actuated carbon nanotube: a dynamical integrity perspective," Nonlinear Dynamics, DOI 10.1007/s11071-013-0986-5, Vol. 74, pp. 533-549, 2013.

[40] L. Ruzziconi, M. I. Younis, and S. Lenci, “An electrically actuated imperfect microbeam: Dynamical integrity for interpreting and predicting the device response," Meccanica, Vol. 48, Issue 7, pp 1761-1775,DOI 10.1007/s11012-013-9707-x, 2013. [41] Alsaleem, F. M., Younis, M. I, and L. Ruzziconi, “An Experimental and Theoretical Investigation of Dynamic Pull-in in MEMS Resonators Actuated Electrostatically," Journal of Microelectromechanical Systems, Vol. 19, Issue 4, pp. 794 - 806, 2010. 


\section{List of figures}

Figure 1: A cross-sectional view of the fabricated microbeam.

Figure 2: A top view picture of the fabricated microbeam and the actuation pad.

Figure 3: Experimental setup used for testing the MEMS device.

Figure 4: Schematic of the doubly clamped microbeam with the half electrode.

Figure 5: A 3-D map of the microstructure profile as seen from the top.

Figure 6: Static deflection of the beam midpoint with the DC voltage until pull-in.

Figure 7: Frequency response to white noise actuation signal at $V_{D C}=30 \mathrm{~V}, V_{A C}=50 \mathrm{~V}$, and at 4 mTorr chamber pressure.

Figure 8: Frequency response curve showing almost linear behavior at $V_{D C}=20 \mathrm{~V}, V_{A C}=5$ V.

Figure 9: Frequency response curve showing hardening behavior at $V_{D C}=25 \mathrm{~V}, V_{A C}=5 \mathrm{~V}$.

Figure 10: Frequency response curve showing a hardening then softening behavior (mixed response) at $V_{D C}=35 \mathrm{~V}, V_{A C}=5 \mathrm{~V}$.

Figure 11: Frequency response curve showing a hardening then softening behavior (mixed response) at $V_{D C}=40 \mathrm{~V}, V_{A C}=5 \mathrm{~V}$.

Figure 12: Frequency response curve showing a softening behavior at $V_{D C}=45 V, V_{A C}=5$ V. 


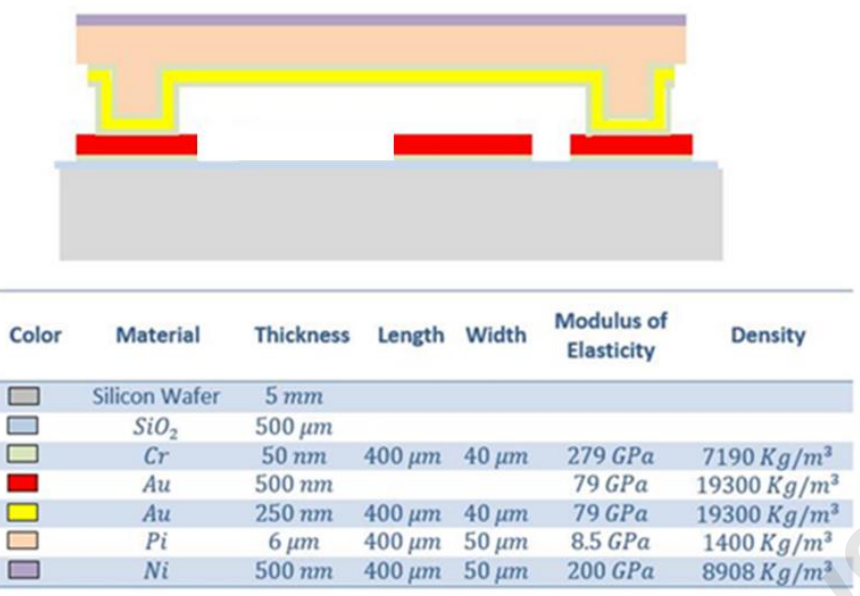

Figure 1: A cross-sectional view of the fabricated microbeam. 


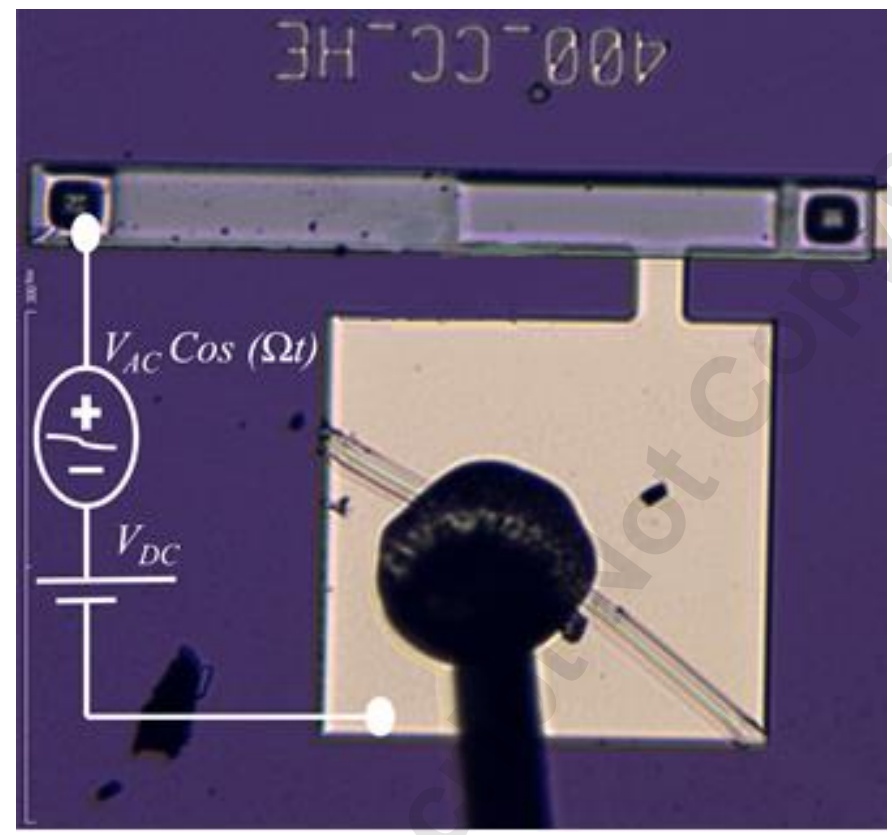

Figure 2: A top view picture of the fabricated microbeam and the actuation pad. 


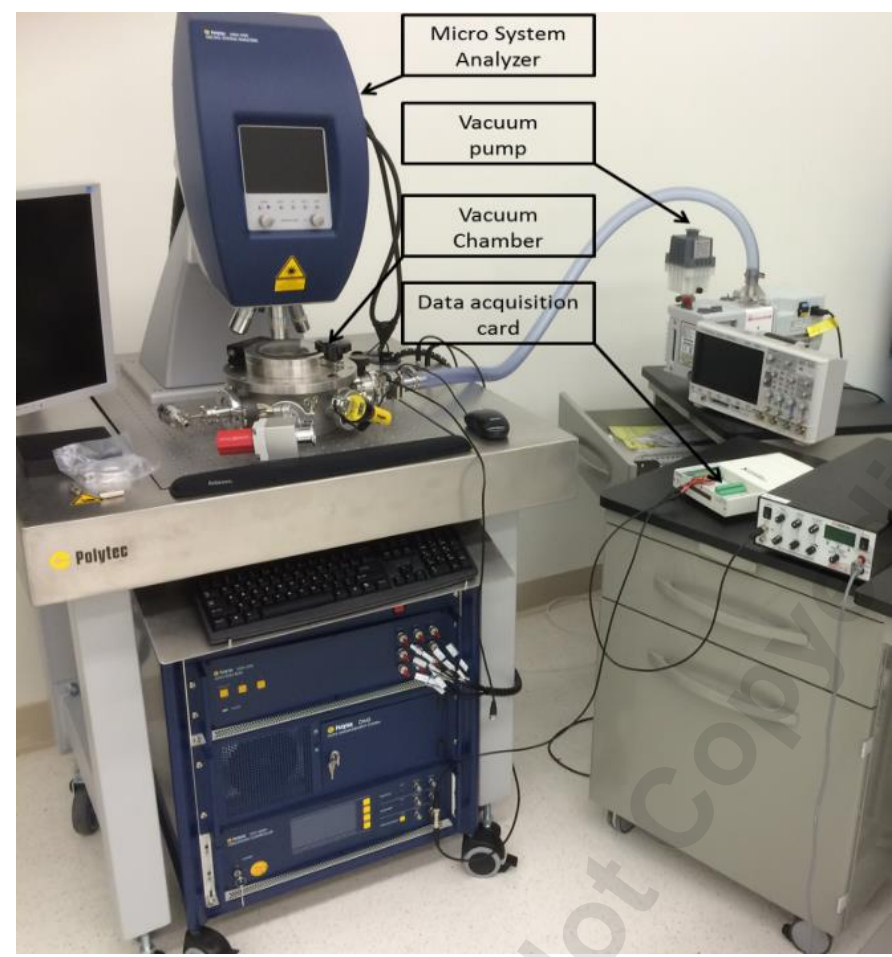

Figure 3: Experimental setup used for testing the MEMS device. 


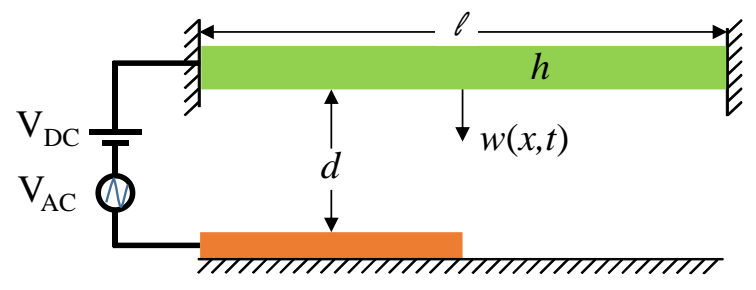

Figure 4: Schematic of the doubly clamped microbeam with the half electrode. 

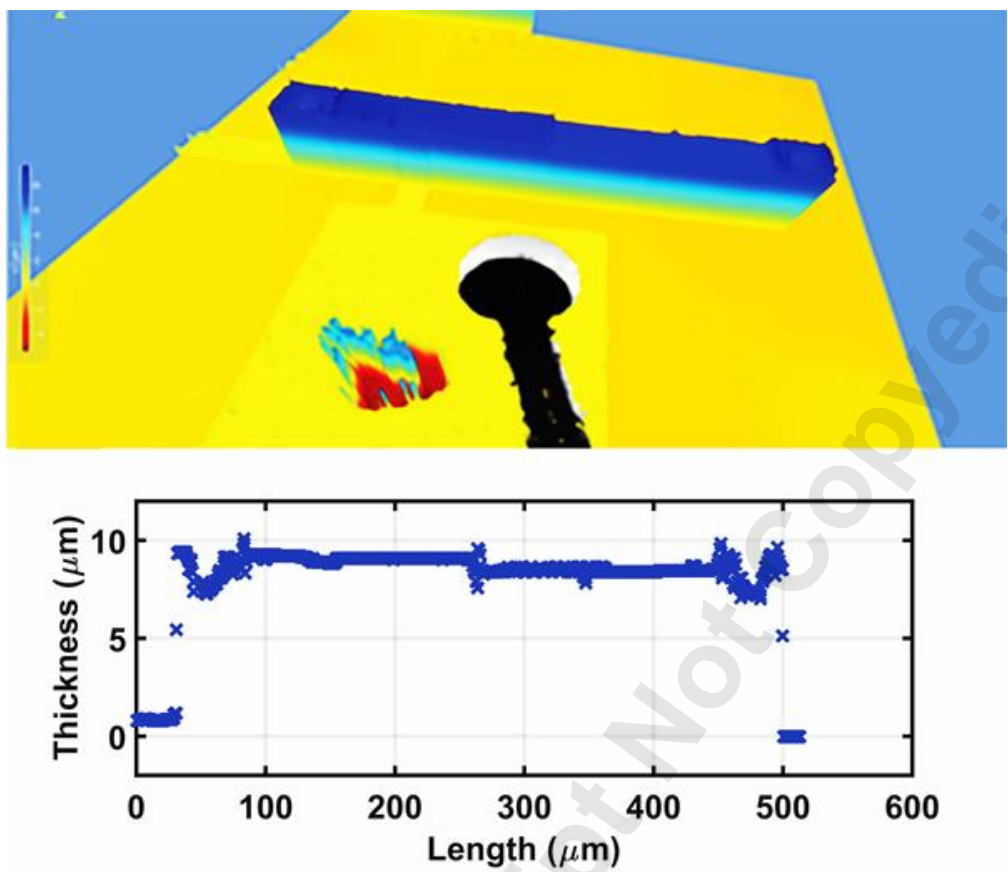

Figure 5: A 3-D map of the microstructure profile as seen from the top. 
Copyright (c) 2017 Journal bf Vibration and Acoustics on the Dynamics of MEMS and NEMS

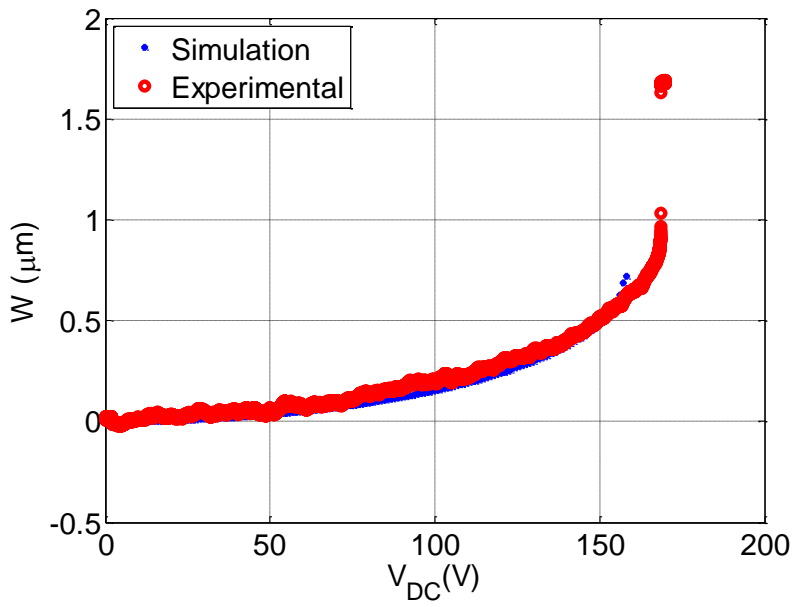

Figure 6: Static deflection of the beam midpoint with the DC voltage until pull-in. 


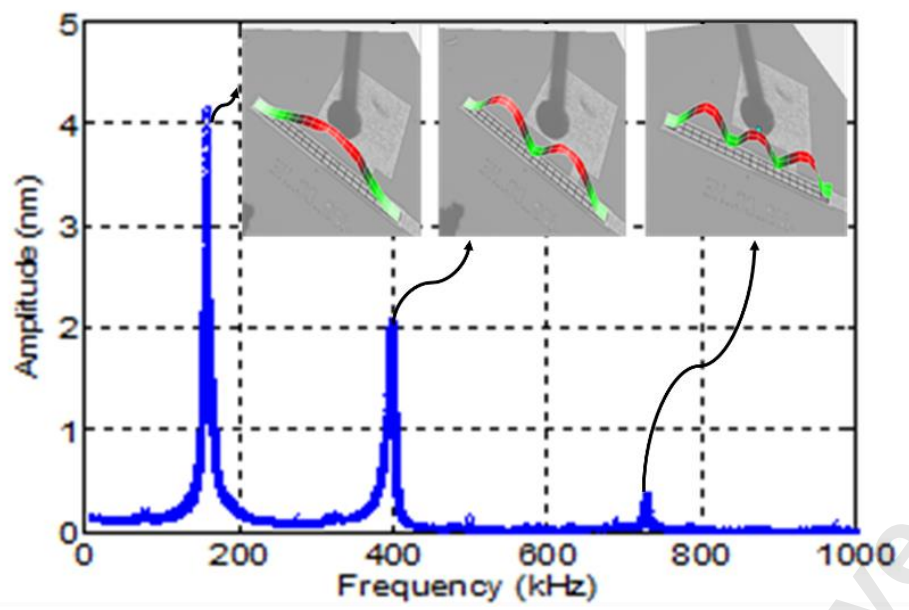

Figure 7: Frequency response to white noise actuation signal at $V_{D C}=30 \mathrm{~V}, V_{A C}=$ $50 \mathrm{~V}$, and at 4 mTorr chamber pressure. 


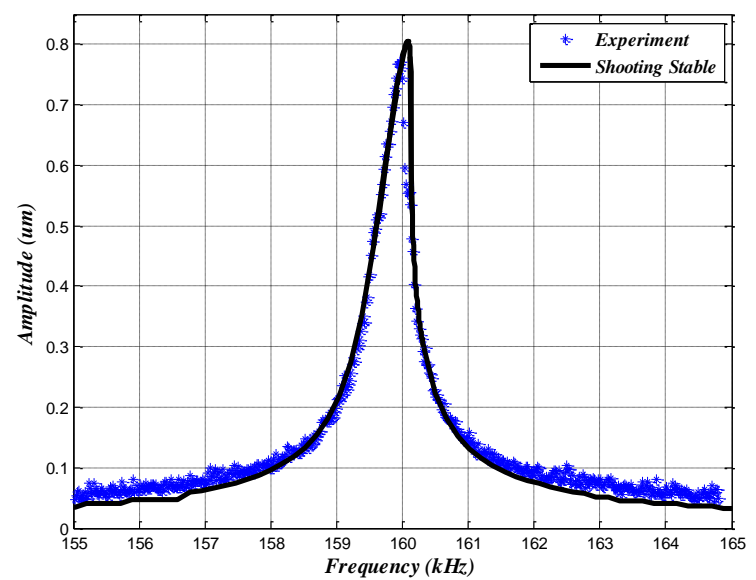

Figure 8: Frequency response curve showing almost linear behavior at $V_{D C}=20$

$V, V_{A C}=5 \mathrm{~V}$. 


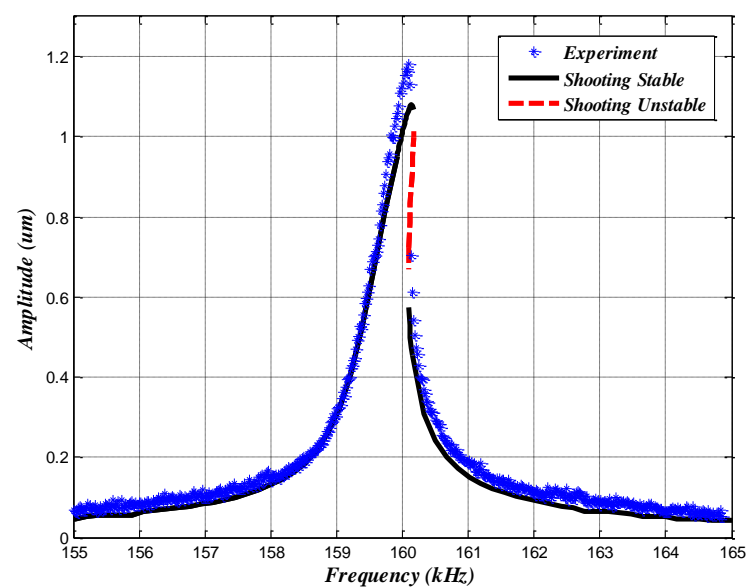

Figure 9: Frequency response curve showing hardening behavior at $V_{D C}=25 \mathrm{~V}$, $V_{A C}=5 \mathrm{~V}$. 


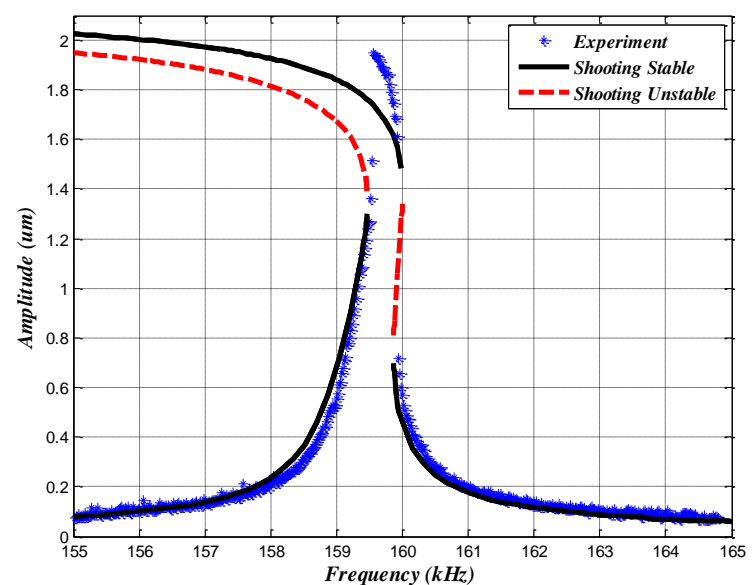

Figure 10: Frequency response curve showing a hardening then softening behavior (mixed response) at $V_{D C}=35 V, V_{A C}=5 \mathrm{~V}$. 


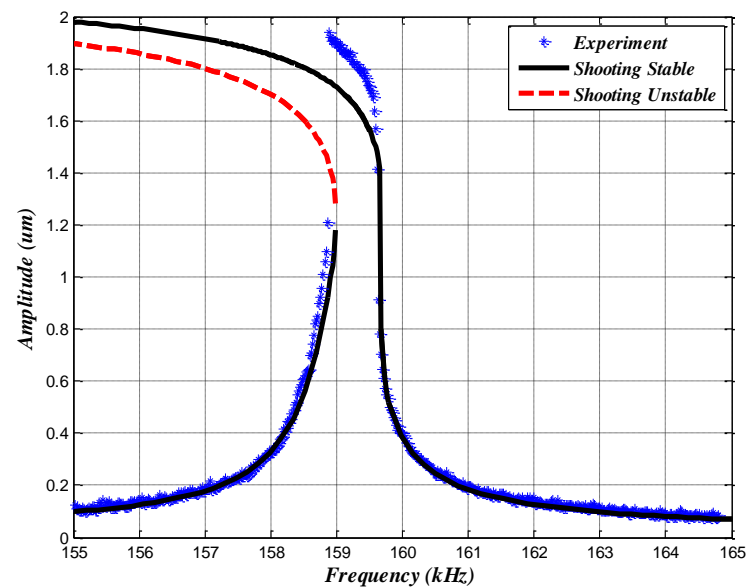

Figure 11: Frequency response curve showing a hardening then softening behavior (mixed response) at $V_{D C}=40 \mathrm{~V}, V_{A C}=5 \mathrm{~V}$. 


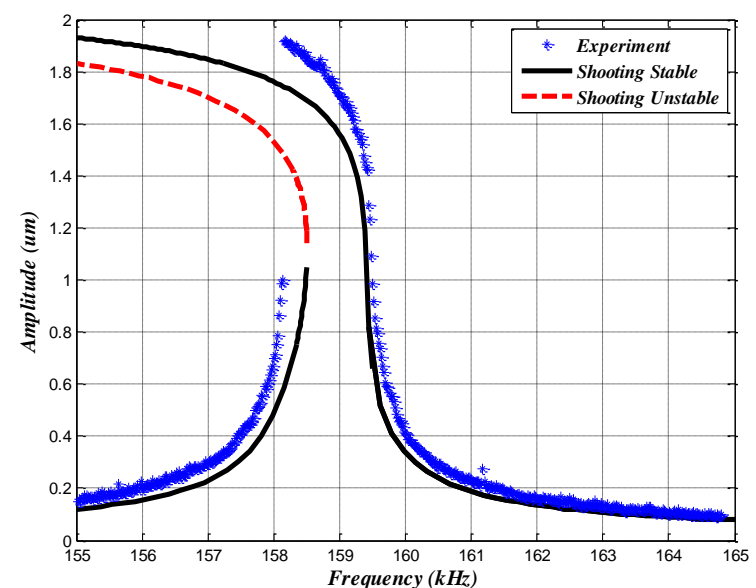

Figure 12: Frequency response curve showing a softening behavior at $V_{D C}=45 \mathrm{~V}$, $V_{A C}=5 \mathrm{~V}$. 